NASA Technical Memorandum 107438

\title{
Direct Data Distribution From Low-Earth Orbit
}

James M. Budinger, Gene Fujikawa, Richard R. Kunath, Nam T. Nguyen, Robert R. Romanofsky, and Rodney L. Spence

Lewis Research Center

Cleveland, Ohio

Prepared for the

International Conference on Communications

sponsored by the Institute of Electrical and Electronics

Engineers Communications Society

Montreal, Canada, June 8-12, 1997

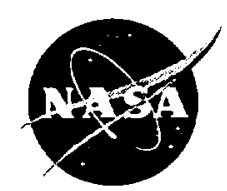

National Aeronautics and

Space Administration 
Trade names or manufacturers' names are used in this report for identification only. This usage does not constitute an official endorsement, either expressed or implied, by the National Aeronautics and Space Administration. 


\section{Direct Data Distribution From Low-Earth Orbit \\ James M. Budinger, Gene Fujikawa, Richard R. Kunath, \\ Nam T. Nguyen, Robert R. Romanofsky, and Rodney L. Spence \\ National Aeronautics and Space Administration \\ Lewis Research Center \\ Cleveland, Ohio}

ABSTRACT: NASA Lewis Research Center (LeRC) is developing the space and ground segment technologies necessary to demonstrate a direct data distribution $\left(D^{3}\right)$ system for use in space-to-ground communication links from spacecraft in low-Earth orbit (LEO) to strategically located tracking ground terminals. The key space segment technologies include a K-band (19 GHz) MMICbased transmit phased array antenna, and a multichannel bandwidth- and power-efficient digital encoder/modulator with an aggregate data rate of $622 \mathrm{Mb} / \mathrm{s}$. Along with small (1.8 meter), low-cost tracking terminals on the ground, the $D^{3}$ system enables affordable distribution of data to the end user or archive facility through interoperability with commercial terrestrial telecommunications networks. The $D^{3}$ system is applicable to both government and commercial science and communications spacecraft in LEO. The features and benefits of the $D^{3}$ system concept are described. Starting with typical orbital characteristics, a set of baseline requirements for representative applications is developed, including requirements for onboard storage and tracking terminals, and sample link budgets are presented. Characteristics of the transmit array antenna and digital encoder/modulator are described. The architecture and components of the tracking terminal are described, including technologies for the next generation terminal. Candidate flights of opportunity for risk mitigation and space demonstration of the $D^{3}$ features are identified.

\section{INTRODUCTION}

Since the early 1980's, NASA's Lewis Research Center (LeRC) has sponsored the development of advanced satellite communications technologies to mitigate the risk of their use in commercial space applications. The most significant demonstration of multiple advanced technologies to date has been LeRC's Advanced Communications Technology Satellite (ACTS), launched in September 1993 [1], [2]. As the nation's first experimental Ka-band satellite, ACTS employs multiple channel, time-division multiple access (TDMA) $30-\mathrm{GHz}$ uplinks and time-division multiplexed (TDM) $20-\mathrm{GHz}$ downlinks. Both links use electronically switched spot beams and electronically steered scanning beams. Large (2.2-meter and 3.3-meter) spotbeam reflectors are used onboard the ACTS to reduce the size of the ground terminal reflectors.

Since the mid-1990's numerous domestic and international systems have been proposed for constellations of commercial communications satellites in both geostationary Earth orbit (GEO) like ACTS, and low Earth orbit (LEO) as well. Some of the proposed systems will employ $\mathrm{K}$ - or $\mathrm{Ka}$-band space-to-ground links for communications at hundreds of Megabits per second $(\mathrm{Mb} / \mathrm{s})$ between the spacecraft and terrestrial gateway terminals. The first of these systems is slated to become operational before the turn of the century, with many more to follow in the next decade.

In the same timeframe, a series of both commercial and government remote sensing and science spacecraft in LEO are also planned. Each will be equipped with a variety of high-resolution imaging systems and on-board storage. Some will have to store up to tens of Gigabytes of data before an opportunity to transmit the data becomes available. As well, the International Space Station will collect large amounts of mission video and experiment data that must eventually be delivered to principle investigators and archive facilities on the Earth.

There are several alternatives for data distribution from LEO spacecraft. The first of those is to establish nearly continuous return links through GEO relay satellites 22,000 miles away, such as NASA's Tracking and Data Relay Satellites (TDRS), or future commercial GEO communications satellites. At data rates of tens of $\mathrm{Mb} / \mathrm{s}$ such space-to-space links will typically use mechanically steered reflectors with high gain on the LEO spacecraft to overcome significant free-space path loss. The GEO relay spacecraft must also dedicate a tracking communications system for each supported spacecraft in LEO, or schedule periods where both the LEO spacecraft and the GEO relay satellite are in view of each other and within the tracking capability of both systems. Links via 
commercial LEO and medium Earth orbit (MEO) satellite networks may become a viable option in the future. Direct instrument readout with continuous broadcast to scattered ground terminals around the world at data rates to hundreds of kilobits per second $(\mathrm{kb} / \mathrm{s})$ is another approach. The direct data distribution $\left(D^{3}\right)$ system described throughout this paper and illustrated in Figure 1 , employs advanced component technology in both the space and ground segments, to provide an economically and technically attractive alternative to any of the above approaches.

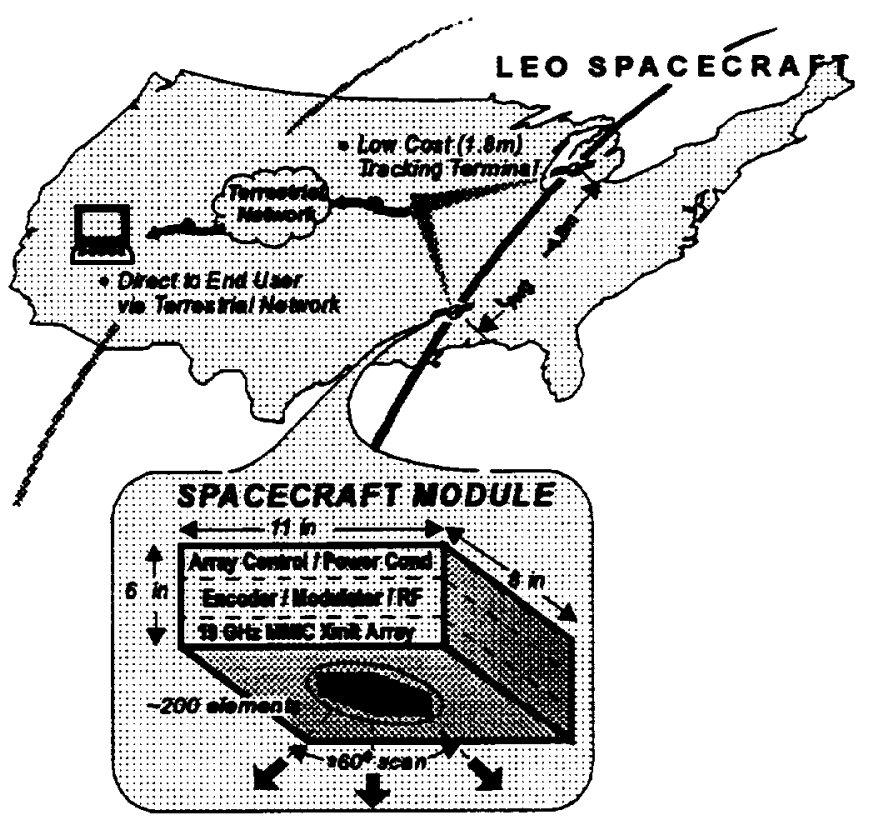

Figure 1 - The Direct Data Distribution System Concept

In the proposed $D^{3}$ system, a small, light-weight package onboard the LEO spacecraft transmits data to strategically placed ground terminals in bursts of data at rates from $155 \mathrm{Mb} / \mathrm{s}$ to $622 \mathrm{Mb} / \mathrm{s}$ as the spacecraft flies though the field of view of the $1.8-\mathrm{m}$ tracking terminals a few hundred miles below. As an example, to equal one $\mathrm{D}^{3}$ downlink burst of an average 4.5-minutes duration at $622 \mathrm{Mb} / \mathrm{s}$ every 90 minutes from a polar-orbiting science spacecraft, the alternatives would have to transmit to LEO or MEO satellites at $31 \mathrm{Mb} / \mathrm{s}$ continuously, or provide sufficient on-board transmit power to overcome about $30 \mathrm{~dB}$ of additional path loss to a GEO relay satellite, or broadcast at about three times that rate to a dense network of ground terminals all around the globe, assuming only one-third of the broadcast time will be over land masses. None of these solutions are practical or cost effective when compared to the $D^{3}$ system concept.

\section{A Convergence of Applications and Technologies}

The $D^{3}$ concept described in this paper represents the convergence of multiple commercial and government applications for high data rate downlinks mentioned above, and multiple advanced technologies that are nearing readiness for system level demonstration. These technologies include: (1) electronically steered, K-band transmit phased array antennas; (2) bandwidth- and power-efficient wideband multichannel digital coded modulation; and (3) low-cost, autonomous tracking Earth terminals with terrestrial network interfaces.

NASA LeRC has demonstrated monolithic microwave integrated circuit (MMIC)-based active phased array antennas operating at both 20 and $30 \mathrm{GHz}$ in various mobile platforms, including an airbome testbed, to demonstrate full duplex communication with the ACTS satellite. These demonstrations have proven conclusively that electronically-steered antennas can be used to close links between a relatively stationary object tens of thousands of miles away (ACTS) and rapidly fluctuating moving platforms. By contrast, in the $D^{3}$ concept, 19$\mathrm{GHz}$ transmit arrays will be used to scan over a range of about \pm 60 degrees with a maximum slant range of less that one thousand miles from a moving, but relatively stable platform. Of especially significant benefit to high resolution science and remote sensing spacecraft, the electronically steered phased array antenna offers the additional advantages of vibration-free operation and reduced deployment complexity compared to conventional mechanically steered reflectors.

NASA LERC has developed prototype digital hardware capable of providing multiple error-correction coding and modulation schemes and data throughput to $155 \mathrm{Mb} / \mathrm{s}$ per channel, with bandwidth efficiency greater than $2 \mathrm{~b} / \mathrm{s} / \mathrm{Hz}$ and power efficiency comparable to the techniques commonly available, such as coded QPSK. Through the use of concatenated codes to provide bit-error-rates comparable to fiber optic cable, burst data throughput equal to Broadband-ISDN and synchronous optical network (SONET) standards, and data formatting compatible with emerging asynchronous transfer mode (ATM) standards, the $\mathrm{D}^{3}$ system enables seamless interoperability with commercial terrestrial wideband telecommunications networks, to optimize further data distribution.

Finally, the use of $\mathbf{K}$-band frequencies pioneered in the United States by NASA LeRC enables a significant 
reduction in the size of tracking terminal antenna reflectors compared to systems operating in X-band. For example, at $19 \mathrm{GHz}$, reflectors can be a factor of 2.3 smaller (all other parameters being equal), compared to their equal gain counterparts operating at $8.4 \mathrm{GHz}$. Lowcost commercially available tracking antenna systems will be integrated with commercial receivers, demodulators, and terrestrial interface equipment to minimize cost and help develop commercial sources for future operational systems. Proposed $\mathrm{D}^{3}$ space flight demonstrations and experiments will downlink one to four independent 155 $\mathrm{Mb} / \mathrm{s}$ channels with an aggregate throughput of 622 $\mathrm{Mb} / \mathrm{s}$, to a 1.8 -meter tracking terminal. The frequency band selected for demonstrating $D^{3}$ system features and performance is from 18.8 to $19.2 \mathrm{GHz}$.

To help reduce the risk of using advanced technologies such as transmit phased arrays and efficient digital modulation in commercial and government applications, NASA LeRC intends to develop hardware models of both and to demonstrate their synergistic performance in space flight of opportunity, such as a Hitchhiker experiment on the Space Shuttle. While these technologies are likely to be used in a variety of applications from commercial LEO satellite communications to scientific or remote sensing spacecraft, this paper will focus on a $D^{3}$ system used in a commercial remote sensing or NASA science data retum application that can tolerate some latency in data delivery. However, similar tradeoffs and analyses are required for a communications satellite-to-gateway application. In the following sections of this paper, the baseline requirements for a composite of the most common LEO science applications that will benefit from $\mathrm{D}^{3}$ will be presented, including link budgets, on-board storage requirements, and ground terminal tracking requirements. The characteristics of the proposed space segment will be described, including the phased array and encoder/modulator, and the features of the tracking ground terminal will be presented.

\section{BASELINE REQUIREMENTS}

Design of a $D^{3}$ system for a specific application involves a tradeoff analysis of many factors. Among these are: (1) the coverage required per spacecraft, which largely determines the number and location of ground terminals; (2) the spacecraft orbit, which determines the time that the spacecraft is in view of a particular ground terminal and the number of passes it will make over the ground station; (3) the amount of stored mission data that needs to be delivered to the ground, which together with the limited ground terminal view time and number of ground terminals determines the data rate that must be employed on the space-to-ground link; (4) the storage capacity onboard the spacecraft - fewer ground terminals means more on-board storage or higher burst rates; and (5) the technical characteristics of the space-to-ground link itself. These determine the maximum data rate on the communications link and how long it can be maintained on a given pass. Important link parameters include the array effective isotropic radiated power (EIRP) and scanning capability of the array; modulation/coding scheme and modem design; frequency band of operation and spectrum available; and ground terminal size and tracking capability. These aspects of a $D^{3}$ system are discussed below.

\section{Space Segment Characteristics}

Key design considerations for the space segment include the spacecraft orbital characteristics, onboard storage requirements, and frequency band of operation.

\section{Orbital Characteristics}

Typical orbits for $\mathrm{D}^{3}$ systems under consideration will have altitudes in the range of 400-800 km (216-432 Nmi). Table 1 below shows some key parameters for these orbits.

Table 1 - Orbital Characteristics for Selected Altitudes

\begin{tabular}{|c|c|c|c|c|c|c|c|c|}
\hline $\begin{array}{l}\text { Altitude } \\
(\mathbf{k m})\end{array}$ & $\begin{array}{l}\text { Period } \\
\text { (min) }\end{array}$ & $\begin{array}{l}\text { Rev/ } \\
\text { dey }\end{array}$ & $\begin{array}{c}\text { Max Time in View } \\
\left(0^{\circ} \text { elevation }\right)\end{array}$ & $\begin{array}{c}\text { Max Time in View } \\
\left(10^{\circ} \text { elevation }\right)\end{array}$ & $\begin{array}{l}\text { Max Time in View } \\
\text { (200 elevation) }\end{array}$ & $\begin{array}{c}\text { Max Angular } \\
\text { Truck Rate ( } \% / \mathrm{sec})\end{array}$ & $\begin{array}{c}\text { Max LOS } \\
\text { Velocity } \\
\text { (km/sec) }\end{array}$ & $\begin{array}{l}\text { Sun Sync } \\
\text { Inclination }\end{array}$ \\
\hline 400 & 92.56 & 15.56 & $10.17 \mathrm{~min}$ & $6.21 \mathrm{~min}$ & $4.03 \mathrm{~min}$ & 1.098 & 7.22 & $97.03^{\circ}$ \\
\hline 500 & 94.62 & 15.22 & $11.55 \mathrm{~min}$ & $7.38 \mathrm{~min}$ & $4.93 \mathrm{~min}$ & 0.87 & 7.06 & $97.40^{\circ}$ \\
\hline 600 & 96.69 & 14.89 & $12.86 \mathrm{~min}$ & $8.50 \mathrm{~min}$ & $5.81 \mathrm{~min}$ & 0.72 & 6.91 & $97.79^{\circ}$ \\
\hline 700 & 98.77 & 14.58 & $14.10 \mathrm{~min}$ & $9.58 \mathrm{~min}$ & $6.66 \mathrm{~min}$ & 0.61 & 6.76 & $98.19^{\circ}$ \\
\hline 800 & 100.9 & 14.28 & $15.30 \mathrm{~min}$ & $10.62 \mathrm{~min}$ & $7.50 \mathrm{~min}$ & 0.53 & 6.62 & $98.60^{\circ}$ \\
\hline
\end{tabular}


Note that the time in view is very short and is highly sensitive to elevation angle constraints. Because of the high atmospheric and rain losses at low elevation angles, $\mathrm{K}$-band links are typically designed to operate at $20^{\circ}$ elevation or higher. This reduces the spacecraft maximum time in view to only 4-7 minutes. For many remote-sensing and solar observation missions where uniform lighting or sun interference is a concern, the orbit inclination is selected to make the orbit sun-synchronous. This means the satellite orbital plane rotates or precesses so as to maintain approximately the same orientation with respect to the Sun as the Earth revolves about the Sun. Note that the last column of the table shows that sunsynchronous inclinations are in the $97^{\circ}-99^{\circ}$ range. Sunsynchronous orbits are therefore nearly polar and retrograde (meaning that the satellite has a westward motion rather than an eastward motion).

The limited ground station viewing time means that the space-to-ground link must operate at a relatively high data rate to transfer stored mission data. Data rate requirements will, in turn, drive the ground terminal parameters as well as the minimum spacecraft EIRP. As an example, Figures $2 \mathrm{a}$ and $2 \mathrm{~b}$ show plots of required ground terminal antenna size versus spacecraft array EIRP for $19-\mathrm{GHz} \mathrm{D}^{3}$ links operating at the BroadbandISDN compatible channel rates of $155 \mathrm{Mb} / \mathrm{s}$ (SONETOC3) and $622 \mathrm{Mb} / \mathrm{s}$ (SONET-OC12). The different curves correspond to different orbit altitudes. The plots are for a $10^{-12}$ BER performance using 8-PSK modulation with a rate 5/6 Trellis code concatenated with a ReedSolomon $(255,239)$ block code described in a later section of this paper. They also assume a $\pm 60^{\circ}$ scan of the array, $4 \mathrm{~dB}$ polarization and pointing loss, and $7 \mathrm{~dB}$ of clear sky link margin.

If, for example, we assume a $400 \mathrm{~km}$ orbit and 1.8 meter ground terminal, a 155-Mb/s downlink can be supported using a $35 \mathrm{dBW}$ EIRP array while a $622-\mathrm{Mb} / \mathrm{s}$ link requires a $41 \mathrm{dBW}$ EIRP array. The EIRP values shown are specified at $0^{\circ}$ scan angle. As will be described later, such high power $\mathbf{K}$-band arrays are being developed. Although not shown, a GEO space-to-ground link using these same arrays at these two data rates would require a significantly larger ground terminal, on the order of 40-70 meters, due to an additional $30 \mathrm{~dB}$ of free space path loss.

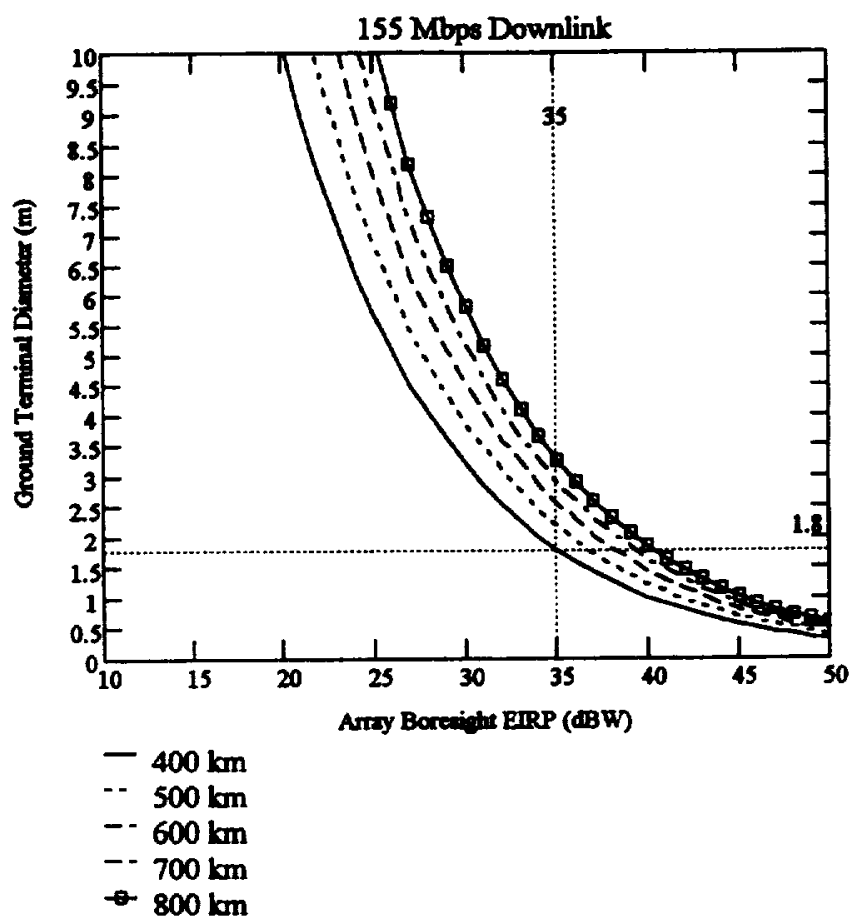

Figure 2a - Ground Terminal Antenna Size versus Array EIRP for $155-\mathrm{Mb} / \mathrm{s}, 19-\mathrm{GHz}$ Downlink at Selected Orbit Altitudes

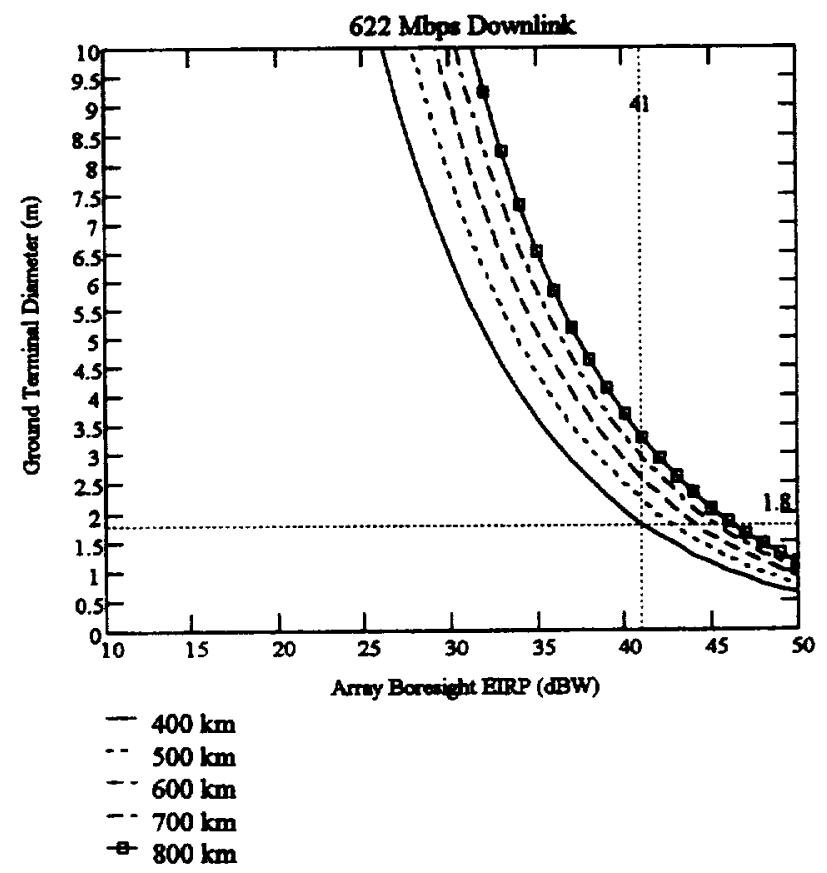

Figure 2b - Ground Terminal Antenna Size versus Array EIRP for $622-\mathrm{Mb} / \mathrm{s}, 19-\mathrm{GHz}$ Downlink at Selected Orbit Altitudes 


\section{On-board Storage}

On-board storage requirements will depend on a number of factors including: the spacecraft orbit; number and location of ground terminals; ground terminal time in view; the rate at which data is generated by the spacecraft instruments; and downlink burst data rate. Instrument raw data generation rates may vary over a wide range from tens or hundreds of $\mathrm{kb} / \mathrm{s}$ for a spectrometer or microwave radiometer to tens or hundreds of $\mathrm{Mb} / \mathrm{s}$ for a synthetic aperture radar or multi-spectral imager. Ground terminal contact time statistics for a given distribution of stations can be found using orbit simulation software. For example, a spacecraft in a 600 $\mathrm{km}$ sun-synchronous orbit $\left(97.8^{\circ}\right.$ inclination) will typically pass over a low to mid-latitude ground station (e.g. Cleveland or Houston) 2-3 times per day and a high latitude station (e.g. Fairbanks) 4-5 times per day.

The average pass duration (not the maximum as listed in Table 1 ) is about 4.5 minutes assuming a $20^{\circ}$ elevation angle mask. At a burst throughput of $622 \mathrm{Mb} / \mathrm{s}$, this leads to a download capacity of almost 168 Gigabits (21 Gigabytes) per pass. For a station in Cleveland, simulation shows that the time between passes can be as much as 13.3 hours (again assuming a $20^{\circ}$ elevation mask) meaning that a typical remote sensing spacecraft which generates data at a $1 \mathrm{Mb} / \mathrm{s}$ rate would require about 48 Gigabits (6 Gigabytes) of storage. A station in Fairbanks, Alaska can experience time between visible passes as large as 11 hours, though frequently the time gaps are only about 1.6 hours ( 1 orbit period). In this case, about 40-Gigabits (5 Gigabytes) of on-board storage are needed. Space-qualified solid state and disk memory devices are available to assemble a storage system to meet such a requirement. Of course, a network of strategically placed ground stations, (e.g. closer to the poles for polar orbiting satellite systems), can allow data to be downlinked more frequently and thus reduce the required on-board storage capacity.

\section{Ground Terminal Characteristics}

\section{Tracking Requirements}

The $\mathrm{D}^{3}$ ground terminal must consist of all the basic subsystems needed to acquire mission data from a spacecraft and its instruments and relay it to end users, either co-located with the terminal, or quite some distance away. One or more ground terminal in a network may also have additional telemetry and command equipment to monitor and control the on-board instruments and payload if this function is not provided through another link to the spacecraft. A variety of solutions are currently available for this function. However, the focus of this paper is the direct data downlink from space in K-band.

In one possible configuration for the outdoor portion of the ground terminal, the antenna subsystem would consist of the reflector and low-noise receiver, the antenna mount, associated tracking positioner, servo motors, and software to control the antenna pointing, and the feeds and transmission lines which carry RF signals to the rest of the RF equipment in the indoor portion of the terminal. For tracking LEO spacecraft, the ground antenna must be able to be steered over essentially the entire visible hemisphere. To minimize losses, communications should only take place when the spacecraft is at least $20^{\circ}$ above the horizon. Antenna pointing may be accomplished either in an open-loop mode using software which predicts satellite ephemeris, or closed-loop mode in which an autotracker uses the received signal itself to steer the antenna. At additional cost, tracking terminals that operate in both modes can be specified.

Table 1 showed that the maximum angular tracking rate required for the ground terminal ranges from about $0.5 \% \mathrm{sec}$ (for an $800-\mathrm{km}$ orbit) to about $1.0 \% / \mathrm{sec}(400-\mathrm{km}$ orbit). The use of higher $\mathrm{K}$-band frequencies places additional demands on the antenna pointing and tracking since the half-power beamwidth (HPBW) is much narrower. For example, a 1.8 -meter dish will have a $0.6^{\circ}$ beamwidth at $19 \mathrm{GHz}$. At S-band frequencies by contrast, the same size dish would have a HPBW on the order of $6^{\circ}$. This means that even if the spacecraft position is known precisely, the $\mathrm{K}$-band ground terminal must still be able to point to within $\pm\left(0.2^{\circ}-0.3^{\circ}\right)$ accuracy to keep pointing loss to $3 \mathrm{~dB}$ or less.

The receive subsystem amplifies and filters the downlink carrier, downconverts it to an intermediate frequency while compensating for Doppler shift, and demodulates and decodes the signal for the data handling and storage system. The maximum Doppler shift will depend on the maximum line-of-sight (LOS) velocity (shown in Table 1) and the frequency. At $19 \mathrm{GHz}$ and orbit altitudes in the $400-800 \mathrm{~km}$ range, maximum Doppler is about 450 to $490 \mathrm{kHz}$. The ground terminal downconverter and demodulator must be designed to acquire and track over this frequency variation. 


\section{Link Budgets}

In a desire to conserve both bandwidth and power resources on the spacecraft, the downlink data is modulated and coded for high spectral and power efficiency. A coded modulation developed by LeRC is envisioned for the proposed $D^{3}$ system. More details on the rate 5/6 trellis-coded 8-PSK modulation with concatenated Reed Solomon $(255,239)$ block coding are described later in the paper. Such a scheme can achieve a
BER of $10^{-12}$ with an $E_{b} / N_{0}$ of $7.2 \mathrm{~dB}$ while transmitting a $155-\mathrm{Mb} / \mathrm{s}$ signal through a $72-\mathrm{MHz}$ channel. The curves in figure 2 assume this modulation and coding method. Table 2 below gives a sample link budget using this scheme for a $460 \mathrm{~km}(250 \mathrm{Nmi})$ orbit and $622-\mathrm{Mb} / \mathrm{s} \mathrm{D}^{3}$ link using a 1.8-meter ground terminal and a $42 \mathrm{dBW}$ EIRP spacecraft array. Note that under clear sky conditions more than $7 \mathrm{~dB}$ of margin is available even at the maximum array scan angle and with $4 \mathrm{~dB}$ of polarization and pointing losses.

Table 2 - Link Budget for 622-Mb/s, 19-GHz Space-to-Ground Link

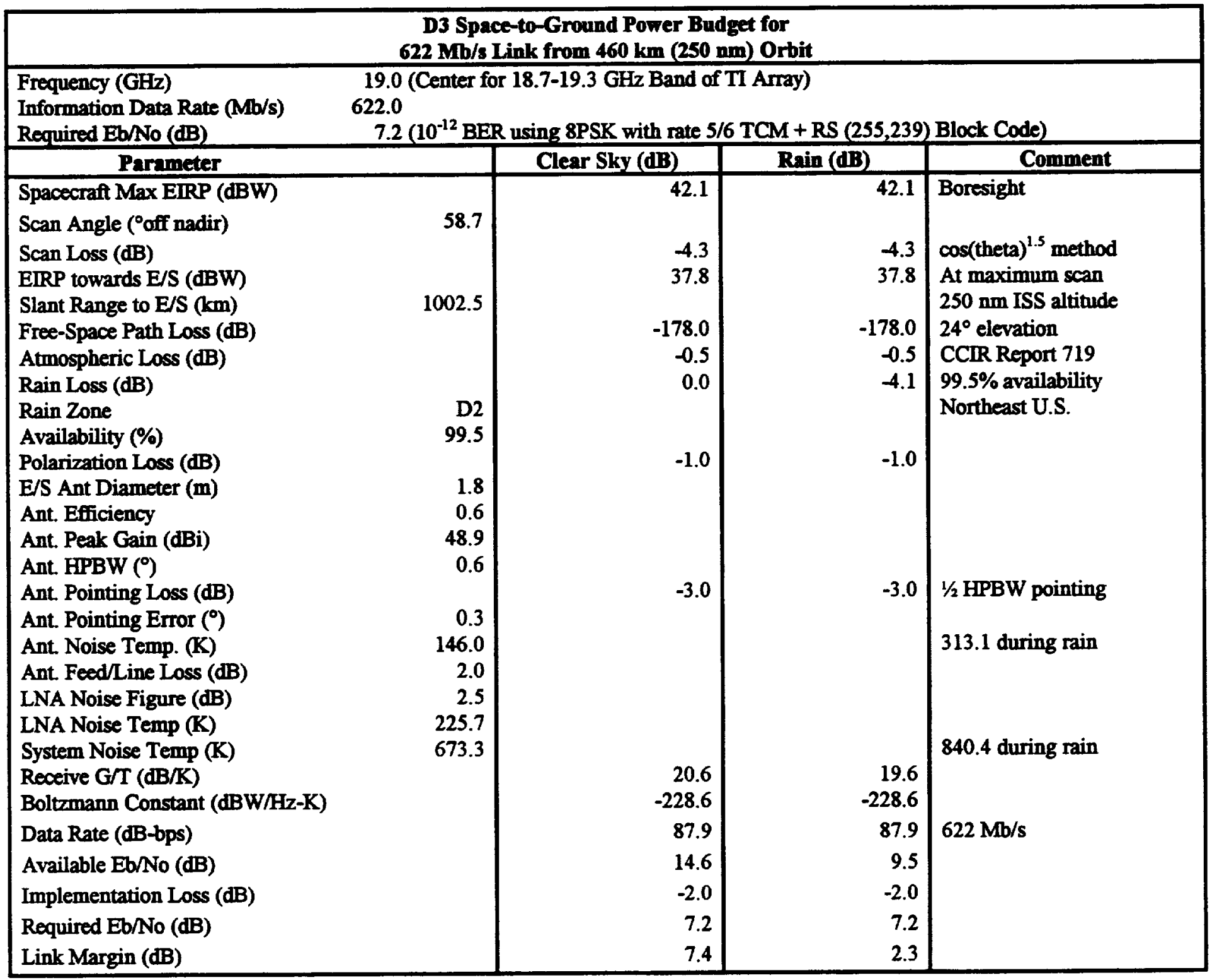




\section{TRANSMIT PHASED ARRAY ANTENNA}

Monolithic microwave integrated circuit (MMC)-based active phased array antennas have been selected for implementation of the space segment. Due to their small size, low mass, high gain, vibrationless electronic scanning, graceful degradation, and conformal profile, active MMIC-based phased array antennas are an ideal candidate for these applications. Active MMIC-based phased arrays eliminate the need for gimbals or spacecraft attitude modification for steering and scanning the antenna beam. The distributed amplification inherent in these antennas mitigates against a catastrophic singlepoint failure, and element weightings can be adjusted to accommodate individual element failures.

\section{Demonstration Heritage}

The active phased array antenna that will be used in a $D^{3}$ space experiment, builds upon the very successful development and demonstration of active MMIC-based phased arrays used in two separate ACTS mobile communications experiments [3]. One demonstrated a link between a NASA Lear Jet and the ACTS, while the other closed a link between an Army HMMWV and the ACTS. Both demonstrations used a 30-GHz transmit tracking phased array antenna that was built under a NASA contract with Texas Instruments (TI) for the uplink to the ACTS.

The TI 30-GHz active phased array was a 32-element array that used 100-mW distributed MMIC amplifiers to generate approximately $22.8 \mathrm{dBW}$ of EIRP. The antenna was capable of electronically scanning \pm 30 -degrees from boresight, with a physical size of $33 \mathrm{~cm}$ by $67 \mathrm{~cm}$. The radiating elements were composed of microstrip, cavitybacked patches with an interelement spacing of $0.8 \lambda(0.9$ $\mathrm{cm})$ [4]. The relatively low EIRP enabled an average link data rate of approximately $20 \mathrm{~kb} / \mathrm{s}$, suitable for low rate data transmission, and ideal for audio (telephonic) transmission, which was the objective of the demonstration; however, slow-scanned video and imagery transmission was also achieved during clear sky transmission times when data rates of $38 \mathrm{~kb} / \mathrm{s}$ were possible.

\section{$D^{3}$ Demonstration Antenna}

The $\mathrm{D}^{3}$ space experiment antenna (supporting an aggregate data throughput of up to $622 \mathrm{Mb} / \mathrm{s}$ from up to four, independently modulated channels) is being developed through a NASA Cooperative Agreement with TI [5]. This array consists of a 224-element active phased array composed of microstrip patch radiating elements. Two different solid-state power amplifiers will be used to generate the required $37.8 \mathrm{dBW}$ of circularly polarized EIRP at the maximum scan angle. The 100 central elements will use $300 \mathrm{~mW}$ amplifiers, while the remaining 124 elements will use $75 \mathrm{~mW}$ amplifiers. This will produce an amplitude tapering across the array which will reduce sidelobe levels. The physical dimensions of the array will be $13.2-\mathrm{cm}$ by $12.9-\mathrm{cm}$, based on an inter-element equilateral-triangular spacing of $0.6 \lambda(0.94-\mathrm{cm})$. This configuration yields a halfpower beamwidth of approximately $6^{\circ}$, at boresight, which translates to an Earth surface beam contour radius of approximately $50 \mathrm{~km}$. The antenna will be limited to a scan angle of $\pm 58.7^{\circ}$ from boresight (nadir) to minimize scan loss and grating lobe visibility while optimizing link duration. The estimated mass of the antenna is $2.3 \mathrm{~kg}$, with an estimated volume of $500 \mathrm{~cm}^{3}$. Figure 3 illustrates the multilayered construction that supports the compact design of the antenna.

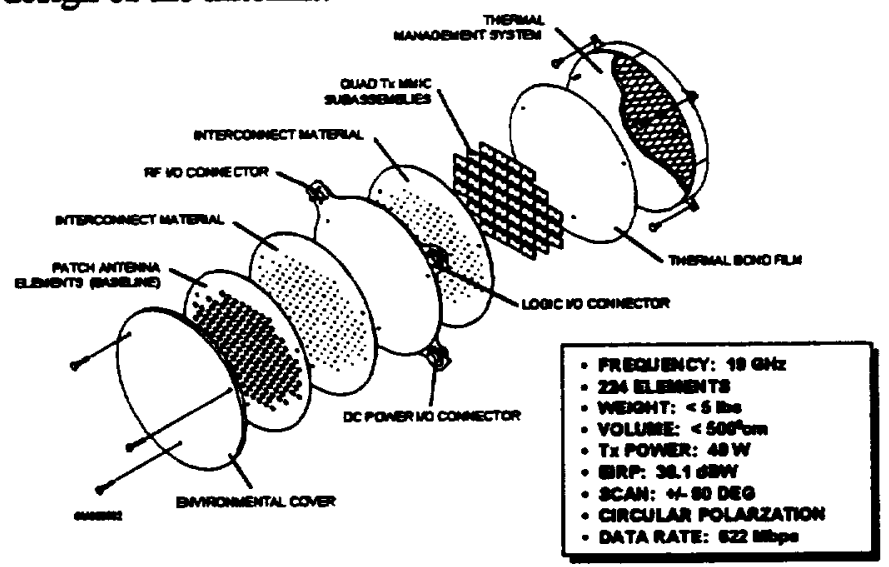

Figure 3 - Direct Data Distribution Array

\section{DIGITAL ENCODER/MODULATOR}

In order to meet the needs of the widest range of $D^{3}$ system applications, a flexible downlink modulation system is needed. NASA LeRC has developed a modular and programmable digital encoder/modulator (DEM) [6], shown in Figure 4 that will serve as the foundation for multichannel implementation in space qualified hardware. The architecture of the DEM provides a level of mission design flexibility allowing the same package to be used on many spacecraft with widely varied communications needs. The DEM provides data rates and quality of service $(\mathrm{Q} O S)$ that is compatible with emerging commercial standards for hybrid spacel terrestrial communications networks such as the 
broadband integrated services digital network (B-ISDN). While allowable error performance requirements for transmission of B-ISDN services over satellite at frequencies above $15 \mathrm{GHz}$ are still being considered in International Telecommunication Union draft recommendations [7] and [8], an end user error rate goal of $10^{-12}$ was selected for the DEM to be used in $D^{3}$ space demonstrations to ensure acceptable error performance.

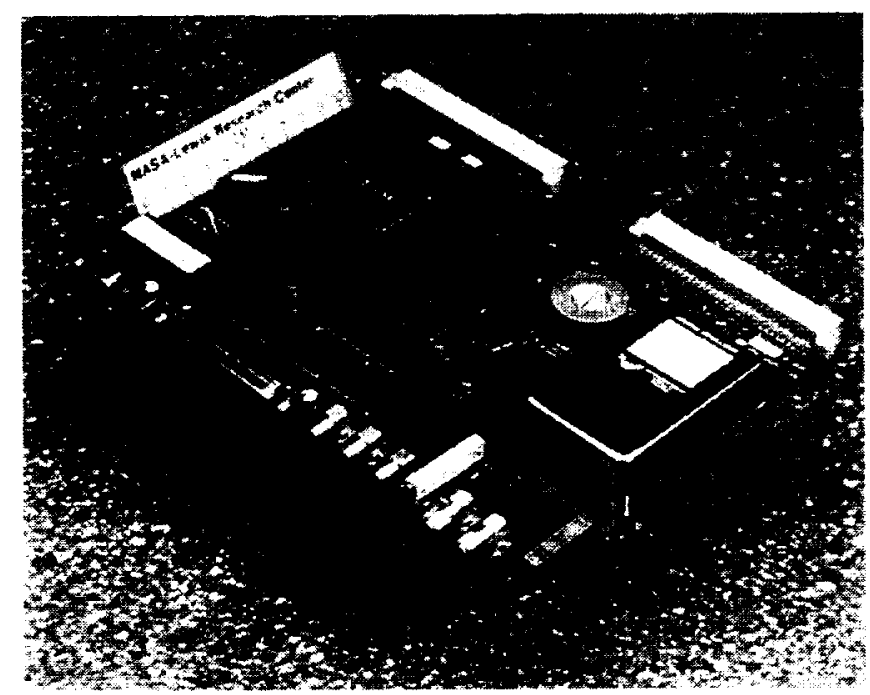

Figure 4 - DEM Hardware

The current single channel prototype DEM hardware supports throughput rates up to $280 \mathrm{Mb} / \mathrm{s}$ and offers several pulse shaped quadrature modulation schemes. These include binary and quaternary phase shift keying (BPSK and QPSK) as well as higher order schemes such as 8-ary PSK (8-PSK) and 16-ary quadrature amplitude modulation (16-QAM). Through software modifications the DEM also supports binary continuous phase modulation (CPM) schemes such as Gaussian minimum shift keying (GMSK); two board configurations can support higher order CPM schemes.

In addition to higher order techniques, combined modulation and forward-error correction coding is used to improve the overall bandwidth and power efficiency. The configuration proposed for $\mathrm{D}^{3}$ space demonstration supports four independent channels, each at the B-ISDN (OC-3) rate of $155.52-\mathrm{Mb} / \mathrm{s}$ contained within $80-\mathrm{MHz}$ of null-to-null bandwidth (commonly referred to as a 72 MHz transponder). The composite signal with $622-\mathrm{Mb} / \mathrm{s}$ total burst rate will occupy $320 \mathrm{MHz}$. This channel bandwidth efficiency is achieved using a Reed-Solomon $(255,239)$ code concatenated with rate $5 / 6$ fourdimensional pragmatic trellis coded modulation (4DPTCM) applied to 8-PSK [9]. As shown in Figure 5, the concatenated coding technique provides $2.34 \mathrm{bits} / \mathrm{symbol}$ and requires $-6.4 \mathrm{~dB} \mathrm{E}_{b} / \mathrm{N}_{0}$ to obtain a BER of $10^{-6}$. Extrapolation yields an estimated $\sim 7.2 \mathrm{~dB}$ to obtain a BER of $10^{-12}$. Digital filtering and upconversion are used to limit the degradation from theory.

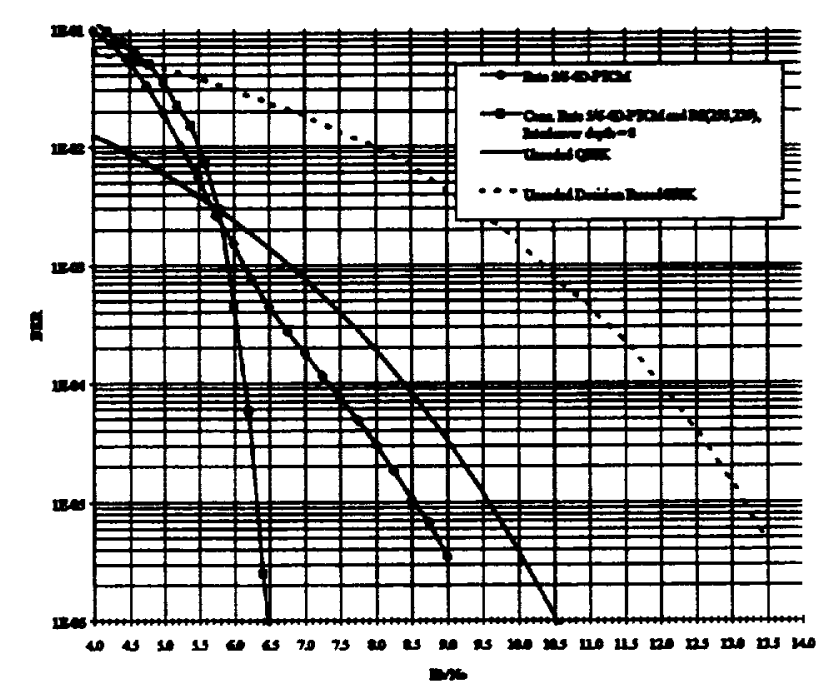

Figure 5 - Baseline Coding BER vs. $E_{\downarrow} / N_{o}$ Performance

As shown in Figure 6, test results show less than $0.2 \mathrm{~dB}$ of degradation from theory in the range of operation. In prototype hardware implementation the DEM is constructed on a standard VME card (6U x $160 \mathrm{~mm}$ ), and it consumes approximately $15-\mathrm{W}$ of $\mathrm{DC}$ power and has a mass of $0.38-\mathrm{kg}$.

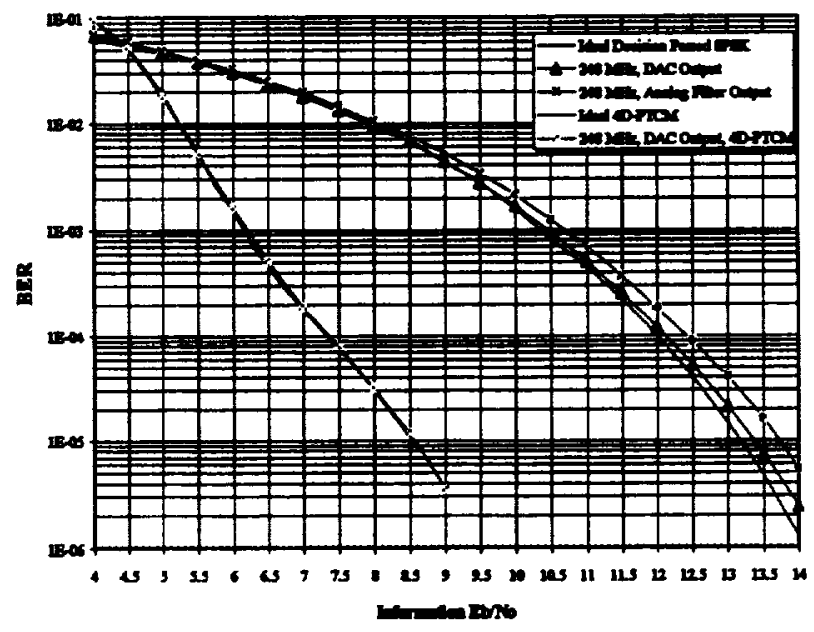

Figure 6 - Hardware and Simulated BER vs. $E_{b} / N_{0}$ Performance

The prototype coded 8-PSK scheme offers good performance in systems that operate through a linear or "linearized" channel. For nonlinear channels, including a 
phased array antenna with individual amplifiers operating in saturation, constant envelope modulation schemes can offer a performance advantage. The DEM architecture has been modified to offer such techniques [10].

For the space based $D^{3}$ demonstrations a version of the DEM that would support the OC-12 data rate of 622$\mathrm{Mb} / \mathrm{s}$ will be developed. One option is the development of an application specific integrated circuit (ASIC) in a high-speed digital process technology, such as ECL, $\mathrm{GaAs}$, or SiGe. Another option is to stack four $155-\mathrm{Mb} / \mathrm{s}$ modulators in frequency, in an orthogonal frequency division multiplexing (OFDM) scheme. While both approaches are currently under investigation at NASA LeRC, the latter affords several benefits including: the ability to fabricate in commercially available radiationhard CMOS technology; lower symbol rate; improved system reliability through an " $m$-for- $n$ " sparing strategy (e.g. 5 on-board encoder/modulators with up to 4 operating at any one time); and operational flexibility to downlink at different aggregate data rates to different classes (sizes) of user terminals, or multiple independent users in the same locale.

SICOM, Incorporated, in collaboration with NASA LeRC, is designing a custom CMOS chip-set rapid acquisition digital data (RADD) modem using DEM technology. The RADD modem will have performance similar to the DEM, but with the reduced size and power consumption afforded by an ASIC implementation. The RADD modem chip-set also contains the demodulator and decoder needed for the low-cost implementation of the ground terminals.

While the coded modulation techniques described above will serve as the baseline approach for a $D^{3}$ demonstration, a modulation study is being conducted in order to determine the effect that the phased array's amplifiers and phase shifters have on a variety of modulation techniques. For example, the operating characteristics of the group of amplifiers (used to boost the EIRP and shape the beam) may largely determine the selection of constant or non-constant envelope modulation. Further, the combined characteristics of the collection of phase shifters (used to steer the beam) are expected to affect the quality of data recovered using $\mathbf{M}$ ary PSK modulation. The latter concern arises because the integer portion of the desired time delay for a specific element in the array is omitted; only the decimal portion is realized in the phase shifter. For example, if the time delay required at the $j$-th element is $n .4$ wavelengths, the $\mathrm{j}$-th element would have 144 degrees of phase shift relative to the reference element instead of $(360 n+144)$ degrees. In large arrays (several wavelengths across the aperture) at wide scan angles, this effect can introduce significant intersysmbol interference (ISI) across the wavefront due to pulse overlap among elements at the extremes of the array. The effect is strongly dependent on symbol rate, carrier frequency, array aperture size, and scan angle. LeRC is beginning to simulate these array-modulation interactions, and is developing a test facility to fully characterize the effects. These results should be used to co-design of the modulation and phased array for improved performance in an operational version of $\mathrm{a} \mathrm{D}^{3}$ system.

\section{GROUND SEGMENT}

The $D^{3}$ ground segment will consist of one or possibly several tracking terminals to handle the large amount of data transmitted from the LEO spacecraft to Earth. Plans are for NASA LeRC to initially develop a prototype receive-only $\mathrm{K}$-band tracking ground terminal for use in a series of timely demonstrations for commercial and NASA applications. Development of these small, low cost tracking terminals can be beneficial in establishing a number of downlink sites in an affordable $D^{3}$ system, such that the effective cost of system operations is reduced significantly in comparison to a network of much larger X-band terminals or LEO-to-GEO relays through NASA's TDRSS. Also, if desired, the small terminals can be co-located with the principle investigator, so that the science data can be retrieved in a faster, more convenient manner than the configuration requiring further data distribution via the commercial terrestrial telecommunications network.

The $D^{3}$ tracking terminal will enable direct data reception at rates up to $622 \mathrm{Mb} / \mathrm{s}$ from the space-based K-band transmit array, and demonstrate terrestrial network interoperability at the highest data rate commercially and readily available at the time of demonstration. The primary goals driving the development of the $D^{3}$ tracking ground terminals are: to minimize cost and ensure commercial transferability; provide an end-user BER of $10^{-12}$ or better; and demonstrate terrestrial network interoperability.

NASA LeRC is leveraging on-going commercial K-band technology development along with its own investment in $\mathrm{K}$-band RF components, antennas, higher order digital modulation and coding described above, and ACTS 
technology to achieve these goals. In an attempt to minimize the cost of the terminals, commercial-off-theshelf (COTS) technology will be utilized to the fullest extent possible.

The tracking antenna system, or TAS, will consist of a 1.8 meter receive-only antenna, a K-band circularly polarized feed assembly with tracking positioner, pedestal, and acquisition/tracking software. The TAS will be capable of acquiring and tracking the various LEO science platforms and spacecraft envisioned in the $\mathrm{D}^{3}$ concept. It will have the ability to acquire and automatically track the satellite during the full time of visibility. A typical satellite pass will be about 4.5minutes, depending on ground elevation angle and satellite altitude. The 1.8 meter parabolic reflector with a circularly polarized feed will operate at a receive frequency band of 18.7 - $19.3 \mathrm{GHz}$. Assuming 55\% aperture efficiency, the gain and half-power beamwidth at 19- $\mathrm{GHz}$ are $48.5-\mathrm{dB}$ and 0.68 -degrees, respectively. The system controller software will utilize orbital element sets (e.g. SGP, SGP-4) to control satellite tracking and antenna positioning. A global positioning satellite (GPS) receiver will be used to maintain an accurate time reference. The positioner can travel 180-degrees in elevation angle and at least 340-degrees in azimuth. The azimuth and elevation velocities are rated greater than 2 degrees per second. The pointing accuracy is specified at \pm 0.1 degrees rms.

The receiver will incorporate all of the RF stages prior to demodulation. Since the composite $622-\mathrm{Mb} / \mathrm{s}$ downlink signal occupies a bandwidth of $320 \mathrm{MHz}$, four (4) RF channels, each requiring a bandwidth of $80 \mathrm{MHz}$, will be preamplified and downconverted to their respective IF bands. The low noise downconverter subsystem will provide the low noise amplification and non-inverting downconversion of the modulated $\mathrm{K}$-band $\mathrm{RF}$ signal. Each IF channel will be bandpass filtered, amplified and distributed to the multichannel demodulator subsystem. A voltage-controlled, tunable local oscillator will allow for a tuning range of $\pm 680-\mathrm{kHz}$ for each channel to compensate for Doppler frequency shifts during each orbital pass.

The multichannel demodulator subsystem for the $D^{3}$ system demonstration will be based on the $155-\mathrm{Mb} / \mathrm{s}$ RADD demodulator currently being developed by SICOM under contract to NASA LeRC. Each single channel demodulator will be modified to operate with decoders compatible with the programmable digital encoder/modulator used in the space segment to meet the BER goal of $10^{-12}$ at $7.2 \mathrm{~dB} E_{b} / N_{0}$.

As previously mentioned, an average orbital pass is about 4.5-minutes. At the downlink rate of $622 \mathrm{Mb} / \mathrm{s}$, the required storage capacity is roughly 21 Gigabytes per pass. Therefore, a Fiber Channel-Arbitrated Loop (FCAL) has been recommended for mass storage. The fiber channel interface loop topology will allow the connection of four (4) disk drives, each with over 8 Gigabytes of storage capability. A dedicated disk drive will be assigned to each of the four demodulators. FC-AL currently provides data transfer rates at 200 Megabytes/second. Data handling and distribution to user or archive facilities will be accomplished via an ATM or other high speed interface.

The terminal system controller (commercially available PC) will provide all required ground terminal control functions. A user-friendly graphical user interface (GUI) will allow for ease-of-use by the system operator. Remote control and monitor of test instrumentation, visual display of satellite track, velocity, altitude, position, terminal health status, schedule orbital pass information, and other pertinent information are to be provided within the various software modules.

\section{Nert Generation Terminal Technologies}

Future versions of the $D^{3}$ terminal will incorporate autonomous operation and commercially compatible high throughput data interfaces to ensure that all received data is delivered to the end user before the next downlink burst arrives. As well, two emerging technologies show promise for future terminal architectures: integrated cryogenic receivers (cryoreceivers) and scanning reflectarray antennas (reflectarrays). Each may offer considerable cost and performance advantages for both the ground and space segment of the $D^{3}$ system.

The physical phenomena responsible for the relationship between the various types of electronic noise and ambient temperature are understood fairly well. Experimental results have shown that the noise temperature of Ka-band pseudomorphic high electron mobility transistor (PHEMT) amplifiers can be rectuced an order of magnitude by lowering the physical temperature of the device from $300 \mathrm{~K}$ to $20 \mathrm{~K}$ [11], [12]. With careful electrical and thermal engineering, some of this advantage can be transferred to the entire receiver such that system noise might be reduced by nearly 6 $\mathrm{dB}$ at the frequency of interest here. Recent advances in 
mochanical refrigeration technology (e.g. Stirling cycle and pulse tube coolers), the discovery of high temperature superconductivity (HTS), and the emergence of GaAs and especially InP PHEMT devices encourage the consideration of cryoreceiver technology. A space-qualified cryoreceiver at X-band has already been developed [13]. Insofar as the current $\mathrm{D}^{3}$ effort is concerned, a $6-\mathrm{dB}$ improvement in receiver system noise temperature could immediately translate into a factor of 4 recuction in the transmitter array EIRP with cascading benefits to other spacecraft systems. Alternatively, one can choose to preserve the $G / T$ of the receiver system and let the antema aperture shrink to about one-quarter of its original area. As a result, the beamwidth would increase by about a factor of 2 . This relaxes the beam pointing requirement and significantly recuces both cost and risk. There may be further advantage because of phase noise rectuction from exploiting HTS resonator stabilized oscillators.

Reflectarrays are being considered for low cost tracking ground terminals. The reflectarray class of antennas was proposed by Berry et al [14] in 1963. A reflectarray consists of a surface containing an array of elemental antennas which are illuminated by a primary feed horn. The array is designed to reradiate the same sense polarization as is incident from the hom, and a cophasal field can be generated in essentially any direction by adjusting the phase shift associated with each element. A beamforming manifold is not required. Hence, the reflectarray combines the best attributes of a parabolic reflector and a direct radiating phased array. That is, a reflectarray provides the electronic beam steering capability of a direct radiating array and is competitive with steerable parabolic reflectors and probably superior to MMIC arrays in terms of manufacturing cost and efficiency. A Ka-band passive 276 spiral antenna element reflectarray is being characterized to determine overall efficiency including spillover, element loss, and crosspolarization effects.

\section{DEMONSTRATIONS AND APPLICATIONS}

NASA LeRC is currently investigating flights of opportunity to demonstrate the $\mathrm{D}^{3}$ concepts and technologies in a space experiment. Using best commercial practices, flight models of the $\mathrm{K}$-band transmit phased array antenna and digital encoder/modulator will be developed for a Hitchhiker class risk mitigation experiment aboard the Space Shuttle. Candidate programs within NASA for application of the $\mathrm{K}$-band $\mathrm{D}^{3}$ approach include New
Millennium near-Earth missions, Space Shuttle, International Space Station, and future Earth Observing System spacecraft. Opportunities for demonstrations in commercial and other government applications are also being investigated, including communications satellite links to terrestrial gateway terminals, and remote sensing data return.

\section{CONCLUSION}

The $D^{3}$ system concept and the enabling space and ground segment technologies have been described. Kband phased array antennas will enable wide bandwidth downlinks from LEO science and communications spacecraft to strategically placed tracking ground terminals. During an average pass of 4.5 mimutes, a D3 system operating at a total burst throughput of $622 \mathrm{Mb} / \mathrm{s}$ will deliver about 20 Gigabytes of data to the ground. Efficient digital modulation and coding will enable quality of service commensurate with that of terrestrial fiber optic cable, with no increase in bandwidth requirements over modulation techniques in common use in today's satellite systems. Ground terminals that are able to acquire and track the LEO spacecraft autonomously will provide an inexpensive alternative to larger X-band systems. The use of standard data rates and formats will enable interoperability with the commercial terrestrial telecommunications network, providing rapid and cost-competitive data distribution to widely distributed end users and archive facilities. For NASA science spacecraft and inhabited space vehicles and platforms in LEO, the K-band $\mathrm{D}^{3}$ concept represents a new paradigm in data delivery systems; one that at once, draws on and enhances emerging commercial communications technologies and services.

\section{REFERENCES}

1. Schertler, R., “ACTS Experiments Program," ACTS Results Conference Proceedings, September 11-13, 1995.

2. Gedney, R., "Results from ACTS Development and On-orbit Operations," ACTS Results Conference Proceedings, September 11-13, 1995.

3. Martzaklis, K. and C. Raquet, "A Mobile Phased Array Antenna Satellite Terminal and Associated Demonstrations with ACTS", AIAA 16th International Communications Satellite Systems Conference, February 1996. 
4. Sanzgiri, S., D. Bostrom, W. Pottenger, and R. Lee, "A Hybrid Tile Approach for Ka-Band Subarray Modules", IEEE Transactions, AP-S, Volume 43, Number 9, pp. 953-959, September 1995.

5. Cooperative Agreement No. NCC3-497, "Development and Demonstration of a $\mathrm{K} / \mathrm{Ka}$ Phased Array Antenna for Direct Data Distribution and Intersatellite Links from Low Earth Orbit".

6. Vanderaar M., D. Mortensen, R. Bexten, N. Nguyen, "A Low Complexity Digital Encoder-Modulator for High Data Rate Satellite B-ISDN Applications," at Milcom 96, McLean Virginia, Oct. 21-24 1996.

7. International Telecommunications Union Recommendation ITU-R S.1062, "Allowable Error Performance for a Hypothetical Reference Digital Path Operating at or Above the Primary Rate", Question ITU-R 75/4, 1994.

8. International Telecommunications Union Draft Recommendation ITU-T 1.356, "B-ISDN ATM Layer Cell Transfer Performance," November 1993.

9. Vanderaar M., R. Bexten, M. Kifle, J. Quintana, “A Concatenated Pragmatic Rate 5/6 Four Dimensional 8-ary Trellis and Reed-Solomon $(255,239)$ Decoder for B-ISDN," currently under NASA review.
10. Vanderaar M., "An Investigation of a M-ary Gaussian Minimum Shift Keyed (MGMSK) Enhancement to the Digital Encoder Modulator (DEM)," Presentation to NASA Lewis Research Center, Oct. 1995.

11. Pospieszalski, M.W., Nguyen, L.D., Lui, M., Liu, T., Thompson, M.A., Delaney, M.J., "Very Low Noise and Low Power Operation of Cryogenic AlInAs/GaInAs/InP HFET's," IEEE MTT-S Digest, 1994, pp. 1345-1346.

12. Lai, R, Bautista, J.J., Fujiwara, B., Tan, KL., Ng G.I, Dia, R.M., Streit, D., Liu, P.H., Fresdenthal, A. Laskar, J., Pospieszalski, M.W., "An Ultra-Low Noise Cryogenic Ka-Band InGaAs/InAlAs/InP HEMT FrontEnd Receiver," IEEE Microwave and Guided Wave Letters, Vol. 4, No. 10, Oct. 1994, pp. 329-333.

13. Javadi, H.H.S., Bowen, J.G.,Rascoe, D.L., Romanofsky, R.R, Chorey, C.M., Bhasin, K.B., "Jet Propulsion Laboratory/NASA Lewis Research Center Space Qualified Hybrid High Temperature Superconducting/Semiconducting 7,4 GHz LowNoise Downconverter for NRL HTSSE-II Program," IEEE Trans. MTT, Vol. 44, No. 7, July, 1996, pp. 1279-1288.

14. Berry, D.G., Malech, R.G., Kennedy, W.A., "The Reflectarray Antenna," IEEE Trans. Ant. Prop., Vol. 11 , Nov. 1963, pp. 645-651. 


\section{REPORT DOCUMENTATION PAGE}

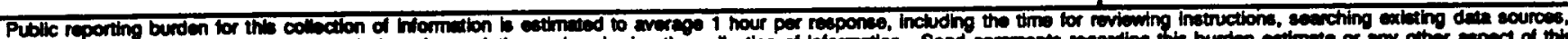

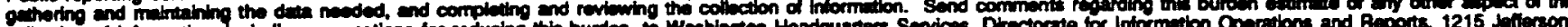

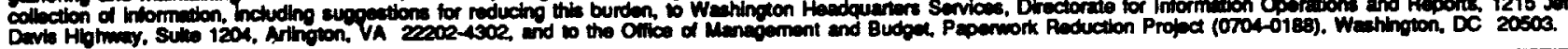

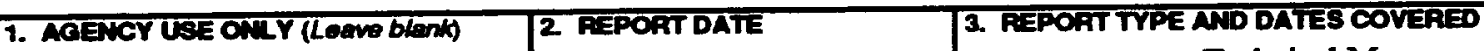

\begin{tabular}{|l|l|l|l} 
April 1997 & Technical Memorandum \\
\hline
\end{tabular}

4. TILE AND SUBTILE 5. FUNDING MUMBERS

Direct Data Distribution From Low-Earth Orbit

WU-315-90-2G

8. AUTHOP(S)

James M. Budinger, Gene Fujikawa, Richard R. Kunath, Nam T. Nguyen, Robert R. Romanofsky, and Rodney L. Spence

7. PEAFOAIING ORGANZATION NAME(S) AND ADORESS(ES)

8. PERFOPINMG OAGANZATION REPOFT MUMBER

National Aeronautics and Space Administration

Lewis Research Center

Cleveland, Ohio 44135-3191

E-10703

9. SPONSORWGMONTOPANG AGENCY MAME(S) AND ADORESS(ES)

National Aeronautics and Space Administration

Washington, DC 20546-0001

10. Sponsomingencontroping

AGENCY REPORT NUIBER

NASA TM-107438

11. SUPPLEMENTAAY NOTES

Prepared for the International Conference on Communications sponsored by the Institute of Electrical and Electronics

Engineers Communications Society, Montreal, Canada, June 8-12, 1997. Responsible person, James M. Budinger, organization code 6150 , (216) 433-3496.

12a. DISTRUBUTOONAVALABIUTY STATEMENT

Unclassified - Unlimited

Subject Categories 17 and 32

This publication is available from the NASA Center for AeroSpace Information, (301) 621-0390.

13. ABSTRACT (Naximum 200 words)

NASA Lewis Research Center (LeRC) is developing the space and ground segment technologies necessary to demonstrate a direct data distribution $\left(\mathrm{D}^{3}\right)$ system for use in space-to-ground communication links from spacecraft in low-Earth orbit (LEO) to strategically located tracking ground terminals. The key space segment technologies include a K-band (19 GHz) MMIC-based transmit phased array antenna, and a multichannel bandwidth- and power-efficient digital encoder/modulator with an aggregate data rate of $622 \mathrm{Mb} / \mathrm{s}$. Along with small (1.8 meter), low-cost tracking terminals on the ground, the $\mathrm{D}^{3}$ system enables affordable distribution of data to the end user or archive facility through interoperability with commencial terrestrial telecommunications networks. The $D^{3}$ system is applicable to both government and commercial science and communications spacecraft in LEO. The features and benefits of the $D^{3}$ system concept are described. Starting with typical orbital characteristics, a set of baseline requirements for representative applications is developed, including requirements for onboard storage and tracking terminals, and sample link budgets are presented. Characteristics of the transmit array antenna and digital encoder/modulator are described. The architecture and components of the tracking terminal are described, including technologies for the next generation terminal. Candidate flights of opportunity for risk mitigation and space demonstration of the $D^{3}$ features are identified.

14. SUBJECT TERIS

Satellite; Communications; Downlink; Space-to-ground; Distribution; Phased arrays; Modulation; Tracking terminals; Low-Earth orbit

15

16. PFICE CODE

A03

\begin{tabular}{|c|c|c|c|}
\hline $\begin{array}{c}\text { 17. SECUATY CLASSIFICATION } \\
\text { OF REPORT } \\
\text { Unclassified }\end{array}$ & $\begin{array}{c}\text { 18. SECURTY CLASSIFICATION } \\
\text { OF THIS PAGE } \\
\text { Unclassified }\end{array}$ & $\begin{array}{c}\text { 19. SECUPIY CLASSIFICATION } \\
\text { OF ABSTRACT } \\
\text { Unclassified }\end{array}$
\end{tabular}

NSN 7540-01-280-5500

Standard Form 298 (Rov. 2-89)

Prescribed by ANSI Sid. 239-18 290-102 